Rev Inves Clin. 2017;69:66-76

\title{
BREAST CANCER INTRA-TUMOR HETEROGENEITY: ONE TUMOR, DIFFERENT ENTITIES
}

\author{
José Esparza-López ${ }^{1}$, Elizabeth Escobar-Arriaga ${ }^{2}$, Santos Soto-Germes ${ }^{3}$ \\ AND MARÍA de JesÚs IBARRA-SÁNCHEZ ${ }^{1 *}$
}

${ }^{1}$ Biochemistry Unit, Instituto Nacional de Ciencias Médicas y Nutrición Salvador Zubirán; ${ }^{2}$ Oncology Center, Hospital Ángeles del Pedregal, Mexico City; ${ }^{3}$ Integral Oncology Center, Servicio de Tumores Mamarios, Hospital Médica Sur, Mexico City, Mexico.

\begin{abstract}
In recent years, it has become evident that intra-tumor heterogeneity of breast cancer is a big challenge for the diagnosis, treatment, and clinical course of tumor-bearing patients. The advances in molecular biology and other technologies have led to the knowledge that a breast cancer tumor is comprised of multiple cellular entities. Here we review the two theories that have been described, trying to explain the origin of intra-tumor heterogeneity: clonal evolution and cancer stem cells. The first one considers that a single cell gives rise to many subpopulations through the accumulation of multiple aberrations, while the cancer stem cells theory foresees a hierarchical tumor evolution where only a few cells with self-renewal capacity give rise to different subpopulations. We also analyze the genetic, epigenetic, and microenvironment contributions to breast cancer intra-tumor heterogeneity. Finally, the clinical and therapeutic impact of intra-tumor heterogeneity on the outcome of breast cancer patients is discussed. (REV INVES CLIN. 2017;69:66-76)
\end{abstract}

Key words: Breast cancer. Cancer stem cell. Clonal evolution. Intra-tumor heterogeneity.

\section{INTRODUCTION}

Breast cancer is one of the most widespread invasive cancers and represents approximately $25-30 \%$ of cancer-related deaths in women ${ }^{1,2}$; it is also the leading cause of death of Mexican women ${ }^{3}$. Currently, breast cancer is recognized as a heterogeneous disease. Since the beginning of the $20^{\text {th }}$ century, the first evidence of breast cancer heterogeneity was described through

\section{Corresponding author:}

*María de Jesús Ibarra-Sánchez

Biochemistry Unit

Instituto Nacional de Ciencias Médicas

y Nutrición Salvador Zubirán

Vasco de Quiroga, 15

Col. Belisario Domínguez, Sección XVI, Del. Tlalpan

C.P. 14080, Ciudad de México, México

E-mail: maria.ibarras@incmnsz.mx clinical observations. Following this period and at the beginning of the $21^{\text {st }}$ century, gene profiling of a large set of breast cancer tumors revealed five different molecular subtypes: luminal A (estrogen receptor [ER] and progesterone receptor [PR] positive), luminal $B(E R, P R$, and epidermal growth factor receptor 2 [HER2] positive), HER2-positive, basal-like, and normal-like ${ }^{4-6}$, confirming a large inter-tumor heterogeneity. The heterogeneity and diversity of the disease have led to a more 
detailed molecular and cellular classification ${ }^{7}$. Recently, breast cancer has been classified in at least 18-21 subtypes, showing further diversity of a largely heterogeneous disease. Each subtype displays a distinct set of histological, pathological, and molecular characteristics $^{8}$. Nevertheless, current patient management is still limited to its clinical characteristics and classic histological analyses of ER, PR, and HER2 ${ }^{9}$.

The prognosis and clinical outcome differs in each molecular subtype, being luminal A the tumors with better prognosis, and basal-like tumors the worst. Taking into consideration that breast cancers are different entities, biomarkers were used to establish specific treatments for each molecular subtype, leading to a change in the way patients are treated. For example, hormonal receptor-positive tumors are treated with endocrine therapy and anti-HER2 treatment is used in HER2-positive tumors. With the advances in the knowledge of the molecular biology and addressing the inter-tumor heterogeneity of breast cancer, it was thought that one of the main challenges of this disease had been overcome. However, despite all the advances in diagnosis and the vast arsenal of therapeutic drugs, recurrence and therapeutic resistance are common challenges in breast cancer; this could be attributed to intra-tumor heterogeneity due to pre-existence of therapy resisting clones or therapy-induced mutations ${ }^{10,11}$. In this article, we review concepts describing breast cancer intra-tumor heterogeneity generated by different mechanisms like genetic mutations and epigenetic changes, and how these mechanisms affect tumor progression and therapy response.

\section{THEORIES OF INTRA-TUMOR HETEROGENEITY}

The concept of intra-tumor heterogeneity has been evolving in the last decades. Currently, tumors are recognized as complex entities with phenotypic and genetic heterogeneity, not only within the primary tumor, but also with marked differences within their metastases. Two theories have been proposed to explain the intra-tumor heterogeneity: clonal evolution and cancer stem cells (CSC) ${ }^{12,13}$. Initially, these concepts were thought to be mutually exclusive, but now they are considered complementary. Both theories consider that tumor is initiated from single cells with multiple molecular alterations that confer an unlimited proliferation potential, and assume that the microenvironment has an effect over tumor evolution. Despite the features in common, these theories have important differences that are discussed below.

\section{Clonal evolution theory}

In 1976, Nowell proposed the clonal evolution theory ${ }^{14}$, describing that a single cell gives rise to tumor growth through a continued accumulation of mutations. The continuous change in the genome (genetic instability) results in a sequential selection of diverse subpopulations with more aggressive phenotypes. Each subpopulation is able to mutate independently, contributing to intra-tumor heterogeneity ${ }^{12,15}$. Two types of clonal evolution have been established: linear evolution and branched evolution (Fig. 1). In the linear evolution, there is a sequential acquisition of mutations, resulting in clones that are fitter than their predecessors. The grade of heterogeneity through linear evolution is low; heterogeneity is only observed when a new clone has not completely outgrown its predecessor (Fig. 1 A). Linear evolution has been observed in acute myeloid leukemia and multiple myeloma ${ }^{16,17}$. In the branched evolution, different sub-clones coexist and evolve simultaneously. This evolution can be analogous to a branching tree where the trunk is the original clone with the founder aberrations and the branches represent the different sub-clones carrying diverse accumulated mutations that are separated geographically. The arising subpopulations conserve the founder aberrations and acquire new phenotypic and genetic features that fit them under selective pressures (Fig. $1 \mathrm{~B}$ ). It is important to mention that mutational, epigenetic, environmental, and selective pressures can affect the clonal evolution by selecting the fittest clones, thus triggering clonal expansions. Clonal evolution most likely proceeds through branching instead of a linear manner, since branching evolution generates greater clonal diversity, hence contributing to heterogeneity. Breast cancer intra-tumor heterogeneity through clonal evolution has been observed in HER2-positive tumors, where different patterns of HER2 amplification have been observed in distinct regions of a tumor ${ }^{18}$.

Furthermore, proliferation and resistance to a given drug follow Darwinian rules where the most fit clones will progress. Therefore, tumor progression or drug resistance depends on population size, mutation rate, 
Figure 1. Clonal Evolution. This theory considers that a tumor evolves through a continued accumulation of genetic and epigenetic aberrations. A: Linear Evolution. There are sequential acquisitions of mutations giving rise to clones tighter than their predecessors. B: Branched evolution. Different sub-clones exist at the same time and evolve independently, accumulating different genetic and epigenetic aberrations. Triangles and stars represent genetic and epigenetic aberrations, respectively.

A. Linear evolution

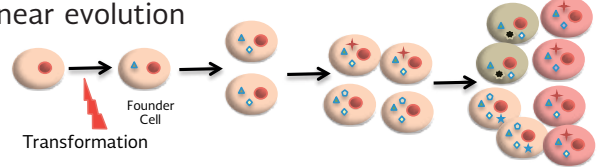

B. Branched evolution

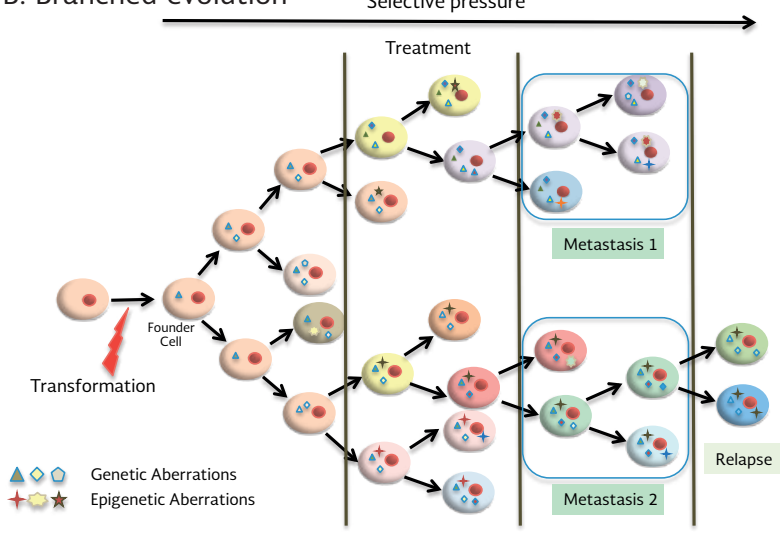

and selective pressures from microenvironment or external factors ${ }^{19}$. Cancer treatment is a common selection pressure that contributes to clonal evolution by selecting clones resistant to a given drug ${ }^{20}$. During tumor growth, subpopulations evolve and acquire mutations that may confer resistance to specific therapies. Once this occurs, resistant subpopulations become the larger proportion of the tumor since susceptible subclones are disappearing ${ }^{21,22}$. In breast cancer, changes in gene expression exerted by neoadjuvant treatment with the aromatase inhibitor (letrozole) have been explored. This study clearly illustrates that neoadjuvant use of letrozole affects proliferation and expression patterns of estrogen-dependent genes ${ }^{23}$. A more recent investigation shows the interplay of clonal evolution and therapy response. Miller, et al. ${ }^{24}$ sampled two time points, before and after four months treatment with letrozole. Using whole-genome sequencing, they characterized the genomic scenery and clonal features of luminal breast cancer, demonstrating that aromatase inhibitor treatment induces remodeling of the clonal populations with the acquisition of new aberrations or enrichment of the pre-existing ones ${ }^{24}$.
Figure 2. Cancer stem cell theory. In this theory, a tumor arises from a rare cell population with self-renewal capacity. Cancer stem cells are characterized by their plasticity to differentiate into non-cancer stem cells and dedifferentiate under specific stimuli. Triangles and stars represent genetic and epigenetic aberrations, respectively. CSC: cancer stem cell.

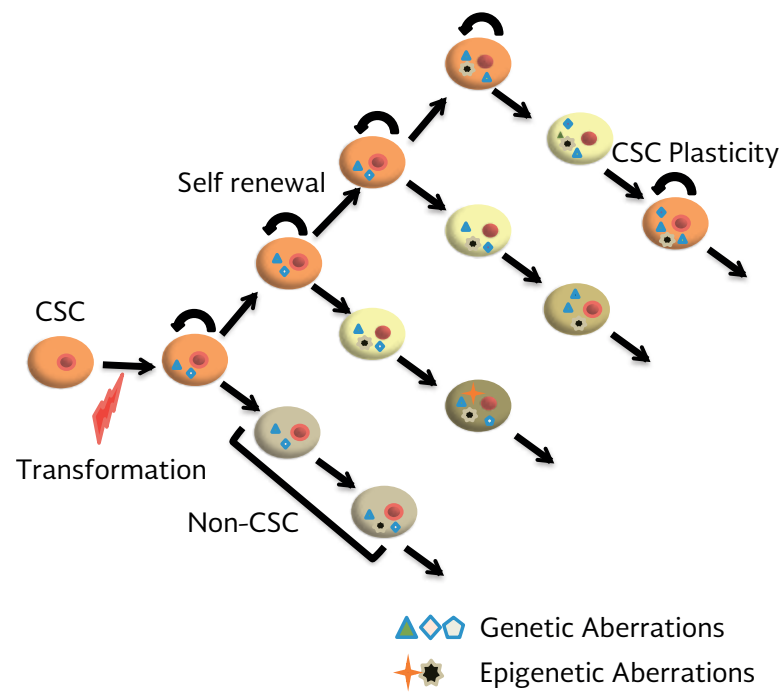

\section{Cancer stem cell theory}

In contrast to the clonal evolution model that claims that all cells within the tumor have the same potential to promote tumor progression, the CSC theory proposes that a tumor arises from a rare small population capable of self-renewal. The CSCs lose their "stemness" by differentiating into non-CSC phenotype, thus giving rise to several subpopulations with a new set of markers (Fig. 2). This aberrant differentiation capacity of CSCs is considered to be responsible for intra-tumoral heterogeneity ${ }^{25}$. The CSC theory was first demonstrated in hematopoietic tumors; later, CSCs were also identified in solid tumors like breast and brain cancers. A combination of cell-surface markers (CD44+/CD24-/low) is used in the identification of CSCs. In breast cancer, a study demonstrated the existence of CSCs by the injection of about 100 CD $44^{+} /$CD $24^{-/ \text {low }}$ cells in a xenograft, and this small amount of stem cells was able to form a tumor. Furthermore, in breast cancer tissues, CD44+/CD24-/low cells have been detected in a low rate ${ }^{26}$. Furthermore, CD $44^{+} /$CD $24^{-/ \text {low }}$ cells have been isolated from cultured breast cancer cell lines; these cells retain their pluripotency and express the cytoprotective factor survivin and the vascular endothelial factor $\mathrm{A}-\mathrm{C}^{27}$. 
In the CSC concept, the tumor is hierarchically organized; the cells with high capacity of proliferation and self-renewal are placed in the highest order ${ }^{28}$. Other characteristics of the CSCs are their capacity to differentiate into a non-CSC cell, also called plasticity, to promote unrestrained tumor growth (Fig. 2). The CSCS divide asymmetrically, resulting in a self-renewal CSC and non-CSC (a less tumorigenic cell). The latter represents the majority of the tumor mass, but contributes less to tumor growth ${ }^{29}$. High recurrence rate has also been attributed to CSCs that posses an innate ability to resist cancer therapy. Cancer treatment has also been reported to promote plasticity that induces the conversion of a cancer cell to a CSC phenotype. There is an equilibrium between the CSCs phenotype and their non-stem lineage; any imbalance within this equilibrium induces a shift to an enriched CSC that will result in an aggressive phenotype and poor prognosis ${ }^{30}$.

Cellular plasticity is defined as the process where a cell can differentiate or dedifferentiate under specific stimuli. Recent studies have demonstrated that a CSC can not only differentiate into a non-CSC, but that this process is reversible and a non-CSC can switch to a CSC under certain conditions. Therefore, there is equilibrium between the CSCs and non-CSCs, and any disruption in the balance, either by genetic, epigenetic, transcription$\mathrm{al}$, or environmental changes, can influence the balance towards dedifferentiation of non-CSCs to CSC that ultimately will result in a more aggressive phenotype ${ }^{31}$.

Furthermore, several investigations have proposed that epithelial-mesenchymal transition (EMT) plays a role in the development of CSCs. The EMT has been extensively studied since it is one of the main steps during the process of tumor metastasis. However, EMT is not only restricted to tumor evolution; there are three types of EMT. The first type is observed during embryogenesis and organogenesis; the EMT is needed to generate the mesoderm that develops into several tissue types. A second type of EMT is associated to wound healing, tissue regeneration, and fibrosis. The third type of EMT has been implicated in tumorigenesis and metastasis $^{32}$. During the EMT, the epithelial cells present reorganization of their cytoskeleton, lose their tight junction proteins and apicobasal polarity. The loss of epithelial markers is one of the main hallmarks of EMT: decreased expression of E-cadherin, claudins and occludins, and concomitantly increased expression of mesenchymal markers like $\mathrm{N}$-cadherin, small muscle actin, and vimentin. These changes are coordinated by a set of transcription factors that include ZEB1, ZEB2, Twist, and Snail ${ }^{33}$. Several studies have demonstrated the participation of these transcription factors in the process of metastasis by either their overexpression or downregulation, observing changes in cell migration and stem cell formation. For example, Twist overexpression in human mammary epithelial induced stem cell markers ${ }^{34}$ and by knocking down Slug in a murine model, the mammary epithelium maintained a stem-like phenotype ${ }^{35}$. ZEB1 overexpression induces the conversion of non-CSC to $\mathrm{CSC}^{36}$. Some parallels have been found between EMT and CSC phenotype. The CSCs have features indicating that they have undergone EMT. There is enough evidence showing that the EMT is connected with the CSC phenotype, and this knowledge has an important impact in the understanding of breast cancer biology ${ }^{34,37}$.

The EMT is a reversible process and cells can undergo a mesenchymal-epithelial transition (MET); this process is also known as epithelial-mesenchymal plasticity $(E M P)^{38}$. Cancer cells, including breast cancer, can change from epithelial to mesenchymal phenotypes and vice versa. It is believed that once tumor cells reach their metastasis site, they need to undergo MET to recolonize it. EMP has been observed in circulating tumor cells and disseminated tumor cells, since they present mixed epithelial and mesenchymal characteristics ${ }^{39}$. A xenograft model of the breast cancer cell line MDAMD-468 showed increased expression of vimentin in circulating tumor cells, and solid tumors presented features of EMT at the periphery, implicating that the edge of the tumor is ready to disrupt the basal membrane ${ }^{40}$. Mutations in the BRCA1 and BRCA2 genes induce a high risk to develop breast and ovarian cancers. Patients with mutations in these genes have poor prognosis. The BRCA1-mutated breast cancer tumors display a basal phenotype. Recently, the relationship between EMT and BRCA1 has been elucidated. Lim, et al. ${ }^{41}$ showed in a humanized mammary mouse model that mutations in BRCA1 increase the EMT transcription factor Snail-2; as mentioned above, this transcription factor is one of the key regulators of EMT. Interestingly, BRCA 1 is a chaperone of Snail-2 for proteasome degradation. Thus, mutations in BRCA1 predispose to EMT, resulting in breast tumors with an aggressive basal phenotype ${ }^{41}$. Regardless of all the information published, it is still controversial whether EMT induces the generation of CSCs, or CSCs use EMT to differentiate into the progeny that will form the tumor mass. While 
there is still debate on their association, EMT and CSCs both contribute to an aggressive phenotype.

\section{TYPES OF INTRA-TUMOR HETEROGENEITY}

In the intra-tumor heterogeneity, different subpopulations coexist within a primary tumor with different behavior, morphology, and genetic and surface markers; this heterogeneity has been attributed to genetic and epigenetic factors ${ }^{42-44}$. Using massive parallel sequencing, a spatial and temporal heterogeneity was found ${ }^{45-47}$. This heterogeneity represents a big challenge for diagnosis and treatment selection since a single biopsy might not be representative of the heterogeneity present in the tumor as a whole. In recent years, intra-tumor heterogeneity has been widely investigated in an effort to understand its origin and impact on prognosis, recurrence, and response to therapy. Genetic and epigenetic factors regarding intra-tumor heterogeneity are discussed below.

\section{Genetic heterogeneity}

An increase in the number of inherited or acquired aberrations in the genome, such as point mutations, translocations, deletions, amplifications, or aneuploidy, leads to what is called genomic instability, which is one of the hallmarks of cancer ${ }^{48}$. Extrinsic factors such as cigarette smoke, ultraviolet light, and chemotherapy are mutagens that also contribute to genetic instability ${ }^{12}$. The acquisition of driver mutations that induce activation of oncogenic pathways and inactivation of tumor suppressors is responsible for cancer initiation and progression. Tumor evolution follows Darwinian principles, where the most fit subpopulations will survive with different abilities in cell proliferation, migration, and invasion ${ }^{10,14,49}$. In every round of cell division, cancer cells accumulate somatic mutations that apparently are not necessary for selection, so they are called "passenger mutations". These mutations apparently are not relevant, but at a given time point during tumor progression or after selective pressure, either from the tumor microenvironment or the systemic therapy administered, the passenger mutation can become a driver mutation, providing new survival advantages. Thus, intra-tumor heterogeneity is produced through the interaction between genome instability and selective pressures, like changes in the microenvironment, hormonal levels, and external factors (therapeutic interventions). Since selective pressures fluctuate over time, the tumor is subjected to different environments, rendering genetic diversification and resulting in genetically, phenotypically, and epigenetically diverse subpopulations ${ }^{50}$.

Several techniques, such as cytogenetic analysis, chromosomal-based genomic hybridization, and massive parallel sequencing, have been used to unmask evidence of intra-tumor heterogeneity in breast cancer ${ }^{18,46,47,51,52}$. Shah, et al. ${ }^{53}$ demonstrated that triple-negative breast cancer presents a wide and continuous range of genomic evolution. Mutations in TP53 and PI3KCA/PTEN seem dominant early in tumor evolution, while mutations in genes of the cytoskeleton and cell motility/shape occur later in tumor progression ${ }^{53}$. Importantly, subpopulations may be present in different geographical regions within the tumor (spatial heterogeneity) or appear at different times during the evolution of the tumor (temporal heterogeneity) ${ }^{46,47,53}$. Spatial heterogeneity means diverse genetic variations across different regions within a tumor. For example, analysis of TP53 by fluorescent in situ hybridization (FISH) and sequencing shows that TP53 presents genetic heterogeneity in metaplastic breast cancer tumors. In some cases, diverse gene copy aberrations were found ${ }^{18}$. Intra-tumor heterogeneity also has been observed in breast cancer biomarkers (ER, PR, HER2), where tumors with spatial areas will exhibit high or low expression of these biomarkers ${ }^{54-58}$.

Temporal heterogeneity appears during the course of breast cancer evolution. There is evidence that some of the somatic mutations in the primary tumor are maintained in the metastasis ${ }^{59,60}$. However, approximately $31 \%$ of the primary tumors and their metastases differ considerably in gene copy number ${ }^{61}$. Whole genome sequencing of a lobular cancer and its subsequent relapse showed that the primary mutations were enriched in the relapsed tumor ${ }^{51}$. In another example, Nik-Zainal, et al. ${ }^{62}$ analyzed the evolution of 21 breast tumors; they found that there is a dominant sub-clonal lineage that represents approximately $50 \%$ of the tumor cells.

\section{EPIGENETIC HETEROGENEITY}

Many studies have linked intra-tumor heterogeneity of cancer and metastatic potential to aberrant epigenetic control and signaling. The study of the major epigenetic enzymes that control chromatin structure and function in breast cancer, including DNA 
methyltransferases, lysine methyltransferases and demethylases, protein arginine methyltransferases, histone acetyltransferases, and deacetylases, have become a major target of research to explain tumor heterogeneity based on epigenetic rearrangements. From all these studies, drugs that target these epigenetic enzymes are being developed rapidly and/or undergoing clinical trials ${ }^{63,64}$. In the following paragraphs, we will discuss the heritable states of gene expression that are not attributed to DNA sequence, but to the process of DNA methylation, a well-known epigenetic mark.

Current therapies have had adverse effects and poor efficacy, prompting the need to find new causes, mechanisms, and biomarkers for breast cancer ${ }^{65,66}$. Prominently, genetic alterations are involved as we discussed above; however, they do not provide a complete explanation for the spectrum of breast cancer heterogeneity. Only $15 \%$ of breast cancer cases have a family history or defined genetic mutations ${ }^{67}$. In this context, epigenetic modifications of chromatin might play a significant role in patients with non-hereditary or non-familiar history of breast cancer. This epigenetic machinery not only has discriminant or prognostic utility, but also could represent therapeutic targets in selected patients ${ }^{68}$. These epigenetic enzymes control breast cancer carcinogenesis, influencing proliferation, invasion, angiogenesis, and immortalization.

Epigenetic regulation refers to changes in genome function that occur without a detectable modification in DNA sequence ${ }^{69}$. During this type of regulation, chromatin is chemically modified, resulting in a specific epigenomic phenotype in a tissue or organ. This modification is heritable through every round of cell replication, thus acting to maintain an unchanging gene expression pattern and promoting a given cell state. This pattern is preserved even when the original stimuli are no longer present. DNA methylation strictly fits this definition; that is, a chemical modification where a methyl group is added on the DNA. In human DNA, this occurs predominantly at cytosine residues in a $\mathrm{CpG}$ context $^{70}$. During cell division, DNA methylation is maintained on the parent DNA strand and copied on the new strand. These modifications are important even to maintain transcriptional activities of the genome or transcriptional repression ${ }^{71}$. Unlike DNA methylation, it is less clear whether histone modifications remain after DNA replication; however, they certainly could be re-established to maintain a stable pattern of transcription of normal and cancer cells. The functionality of the above processes lie in the linear positioning and three-dimensional arrangement of nucleosome that constitutes the "packing" component of epigenetics ${ }^{72}$. The result of these positions in association with DNA modifications is the definition of proper boundaries in normal cells. These boundaries separate tightly packaged and repressive nucleosome domains from the more spaced and transcriptionally active nucleosome arrangements ${ }^{73}$. Then, what happen in early tumors? Experimental evidence suggests that these boundaries are lost $^{71,74,75}$. The result is a change in the structural control of DNA replication and unusual transcription of coding and non-coding regions. Abnormalities of DNA methylation, either losses or focal gains, occur at the same time in well-defined megabase regions ${ }^{76}$. Gains arecancer-specific and normally includenon-DNA-methylated $\mathrm{CpG}$ islands located in large regions of proximal promoters. These changes can be related to loss of gene expression, very similar to inactivating mutations on the abolition of tumor suppressor gene function through linked gene silencing; however, the activation of oncogenes also may occur ${ }^{77}$. In addition to DNA methylation, chromatin abnormalities are also observed in various types of tumors ${ }^{78}$.

\section{Breast cancer epigenetics}

Inherited or acquired mutations in the genome are recognized to be key contributors to the development of breast cancer. Even when family history is the strongest risk factor for developing breast cancer (e.g., germline mutations in the BRCA1, BRCA2, and TP53 genes), other inherited mutations and genetic variations have been identified ${ }^{79}$. The classical view of cancer, including breast cancer, where defects in the DNA repair machinery due to mutations in BRCA1, BRCA2 and other repair genes accelerate the rate of randomly occurring mutations, highlights cancer as a genetic disease. However, epigenetic changes along with genetic mutations are also recognized as important contributors to the development of cancer ${ }^{80}$. Evidence supporting the participation of epigenetic changes in the promotion of cancer has appeared in the literature since the early $1990 \mathrm{~s}^{81}$. Genes involved in chromatin remodeling, like $A R I D 1 A$, or genes that are involved in establishing and maintaining epigenetic patterns (IDH1, MLL3 and KMT2) were described with acquired mutations ${ }^{82,83}$. The BRCA1 gene is frequently inactivated by epigenetic modifications in breast cancer ${ }^{84}$. 
Given that BRCA1 is recognized as an important susceptibility gene, it can be assumed that both epigenetic modifications and mutations participate in the initiation of an oncogenic phenotype ${ }^{85}$. Other tumor-suppressor genes, such as CDKN2A and PTEN, undergo CpG island promoter methylation, but in normal cells, the promoter region is unmethylated ${ }^{86,87}$. Epigenetic studies have revealed that breast cancer subtypes display different methylation profiles and are epigenetically distinct ${ }^{88}$. Evidence has been found on CpG methylation phenotype within luminal subtypes, whereas basal-like subtype seems to have low-grade CpG methylation. Another factor contributing to increased cancer heterogeneity is the involvement of epigenetic changes as well as genetic mutations in breast cancer. Breast cancer genomes usually contain thousands of genetic aberrations, of which only a few actually drive the progress of the disease ${ }^{89}$. In breast cancer, this occurs in tumors carrying mutations in BRCA1 and BRCA2 genes. In general, the loss of the second wild-type allele is an important event leading to breast cancer development. Hypermethylation of the $\mathrm{CpG}$ promoter island is rarely found as a second hit in familial BRCA1 or BRCA2 mutated cancers, while in other tumor suppressor genes (CDKN2A, FZR1, RARB2, GSTP1), hypermethylation is found at a similar rate than in cancer with familial genetic alterations. Thus, epigenetic changes are needed in most cancers, including breast cancer, with DNA repair dysfunction to promote tumor progression. Nevertheless, other genes like TP53, with a high prevalence of mutations in breast cancer and other types of tumors, have no reported $\mathrm{CpG}$ island promoter hypermethylation or other types of suppressive epigenetic modifications ${ }^{90}$. Silencing of the ER along with inactivation of $B R C A 1$ and $B R C A 2$ occur through methylation ${ }^{91}$ and other epigenetic mechanisms. The frequencies of this epigenetic involvement can vary from 17 to $60 \%{ }^{92}$. Similarly, RASSF $1 A$ and HIN1 were found to be hypermethylated in $65-85 \%$ of breast tumors ${ }^{93,94}$. A meta-analysis of nine studies that included a total of 3,205 patients reported that hypermethylation of the BRCA1 gene is associated with a poor survival of breast cancer patients ${ }^{92}$. Nevertheless, most reports agreed with the fact that methylation does not occur in BRCA2, although this is still debatable ${ }^{95}$. In contrast, in sporadic breast cancer there is strong evidence that BRCA1 methylation contributes to tumor progression and aggressive phenotype. It has been demonstrated that primary breast cancer cells with BRCA1 gene defects tend to be poorly differentiated. This undifferentiated phenotype also lacks expression of estrogen and progesterone receptors, but expresses basal-like markers such CK5/6 and epidermal growth factor receptor (EGFR) ${ }^{96}$.

\section{TUMOR MICROENVIRONMENT AND INTRA-TUMOR HETEROGENEITY}

Many of the studies discussed above refer to genetic and epigenetic changes in the tumor cells. However, a tumor mass is not only comprised by neoplastic cells, but is rather a complex network of cells, soluble factors, immune cells, vasculature cells, stromal cells, and extracellular matrix that together constitute the tumor niche or microenvironment. Paracrine signals impact as much as tumor cells and other components on the tumor microenvironment, coevolving as a whole. The microenvironment surrounding the tumor cells influences both genetic and compositional heterogeneity ${ }^{97}$. There is increasing evidence that each component of the tumor niche drives tumor heterogeneity. For example, stromal cells harbor an aberrant extracellular matrix that promotes malignancy and intra-tumor heterogeneity ${ }^{98}$. Stromal cells also secrete factors that induce EMT on epithelial cancer cells ${ }^{99}$. Likewise, tumors with hypoxic regions due to poor vascularization have undifferentiated stem-like tumor cells that survive under the action of hypoxia-inducible factors (HIF) transcription factors family, apparently by inhibiting cell differentiation and maintaining stem-like phenotype ${ }^{100}$.

The immune system plays an important role in cancer progression; it plays a dual role by eliminating cancer cells, but also can stimulate tumor growth. Cancer cells upregulate survival pathways to promote immune suppression and angiogenesis ${ }^{101}$. Moreover, tumor cells possess a high capacity to evade the immune system by different mechanisms. Some cells downregulate the expression of HLA class I, then becoming invisible for cytotoxic T lymphocytes. Through another mechanism, tumor cells secrete factors such as interleukin-10 (IL10) and transforming growth factor-beta that inhibit CD8+ T-cells and induce regulatory T-cells ${ }^{102}$. In breast cancer, it has been suggested that tumor infiltration by lymphocytes indicates an antitumor response ${ }^{103,104}$. Also, the amount of tumor-infiltrating lymphocytes is associated with a better outcome in triple-negative and HER2-positive breast cancers ${ }^{104}$. The question that now remains to be answered is whether breast cancer 
is immunogenic. According to many observations ${ }^{105-107}$, breast cancer is immunogenic due to intra-tumor heterogeneity. It has been demonstrated that $30-50 \%$ of breast cancer overexpressed programmed cell death ligand-1 (PD-L1); the binding with its receptor PD-1 on cytotoxic T-cells blocks the antitumor immune response ${ }^{108}$. The wide variety of clones increases the chances to stimulate the immune system. This idea is supported by lymphocyte breast cancer tumor infiltration and better survival, as mentioned above.

In the same order of ideas, the tumor microenvironment has been compared to a non-healing wound due to the relationship between tumor growth and inflammation. Since a couple of decades, the contribution of chronic inflammation in cancer progression has been clearly recognized. Clinical evidence has associated hepatitis C infection with liver cancer and ulcerative colitis with colon cancer ${ }^{109}$. Expression of inflammation markers, such as C-reactive protein, has been associated with risk of breast cancer recurrence ${ }^{110}$. Several cytokines are secreted in the tumor microenvironment by different types of cells; however, macrophages, mesenchymal cells, and immune cells secrete IL-6 and IL-8; these two cytokines are associated with chronic inflammation. High serum levels of IL-6 have been associated with a poor prognosis of breast cancer patients ${ }^{111}$. Interleukin- 6 is also an inductor of EMT, promoting metastasis and a stem-like phenotype ${ }^{112}$. As IL-8 receptor has been found expressed in breast CSCs, it seems that this cytokine stimulates their self-renewal. The secretion of these two cytokines is regulated by the nuclear transcription factor-kB (NF-kB). Many studies have demonstrated that NF-kB is activated in breast cancer tumors ${ }^{113}$; this activation promotes EMT, resulting in a stem-like phenotype ${ }^{114}$. Since NF-kB participates in every aspect of tumor biology, recent data suggest that NF-kB integrates inflammatory signals from the tumor microenvironment to maintain stemness, leading to has a disadvantageous therapeutic effect.

\section{CLINICAL AND THERAPEUTIC IMPLICATIONS OF INTRA-TUMOR HETEROGENEITY}

It is indisputable that intra-tumor heterogeneity has an important impact on breast cancer diagnosis, disease management, and response to therapy. Monitoring temporal and spatial heterogeneity may be crucial for an accurate diagnosis and treatment selection, as discussed above. Multiple sampling at diagnosis and throughout the disease may be vital for a successful patient outcome; however, sometimes due to the tumor localization, it might not be possible to sample all sites of metastases. Nevertheless, noninvasive sampling offers an alternative choice for genetic and epigenetic testing for an accurate diagnosis and a better choice of treatment ${ }^{115}$.

Recently, intra-tumor heterogeneity has been recognized as one of the mechanism of tumor resistance ${ }^{19}$. Chemotherapy resistance represents the main drawback in response to an effective therapy outcome. There are two types of resistance: intrinsic and acquired. The first is the natural ability of cancer cells to evade the therapy. Acquired resistance refers to tumor cells that initially are sensitive to a given drug, but after some time become desensitized. The EMT has been proposed as one of the mechanisms associated to chemotherapy resistance. In breast cancer patients treated with hormonal therapy, it has been observed that the populations surviving letrozole treatment are enriched with CSCs in the presence of a mesenchymal marker like vimentin ${ }^{116}$. Other studies have reported desensitization to paclitaxel and doxorubicin when Snail- 1 and 2 and Twist are overexpressed in breast cancer cell lines ${ }^{117,118}$. Furthermore, re-expression of E-cadherin in mesenchymal breast cancer cell lines sensitizes to doxorubicin ${ }^{119}$. The CSC plasticity and its relation to the tumor microenvironment and immune-related signals also affect the response to treatment. It is well accepted that cancer cells secret inhibitory factors of the immune system. High levels of colony stimulating factor- 1 induce the recruitment of tumor-associated macrophages that suppress the tumor-inhibitory effect of T-lymphocytes and inhibit the cytotoxic effect of taxanes ${ }^{120}$.

Aberrant methylation found in gene promoters is a hallmark of cancer that could be used as a noninvasive biomarker in body fluids, such as blood and plasma, for early detection of breast cancer, rendering the diagnostic test easier and cost-efficient. Many biomarker genes have been proposed and evaluated; nevertheless, in the absence of a unique biomarker having sufficient specificity and sensitivity, a panel of multiple genes should be used ${ }^{121-123}$. Modifications of cancer-related genes could occur at genetic and epigenetic levels, affecting DNA repair. In the absence of 
proper DNA repair, the cell is more prone to become malignant ${ }^{124}$. Genes harboring genetic and epigenetic changes, such as BRCA1 or BRCA2 mutations, predict breast cancer response to specific anticancer drugs such as PARP inhibitors like Olaparib ${ }^{125}$. Another example is the methylation of the ER promoter that is a better predictor of response to tamoxifen than the expression analysis of ER by immunohistochemistry ${ }^{126}$. Also, most cancer genes are not commonly mutated; it is likely that personalized medicine may be applied to a larger number of cancer patients if epigenetic changes along with genetic mutations are considered. In this vein, anticancer drugs leading to the formation of double-strand breaks, such as platinum-based drugs, have been useful in silencing cells harboring BRCA1 or $B R C A 2^{127}$. Poly adenosine-diphosphate [ADP]-ribose polymerases (PARP) inhibitors have also shown their usefulness against cancer cells with defects in DNA repair machinery. In this context and based on the similarities between BRCA1-mutated breast cancers and basal-like phenotype, PARP inhibitors were tested in patients with triple-negative sporadic breast cancers with controversial results ${ }^{128}$. Nevertheless, this supports BRCA1 methylation testing as a good epigenetic marker of response to PARP inhibitors and to other DNA damage drugs, such as platinum-based agents, as well as cisplatin. Selecting these drugs to treat patients with defects in the BRCA1 gene will lead to significant improvements in disease outcome for patients with a triple-negative phenotype.

\section{CONCLUSIONS}

It has become clear that breast cancer is a very complex disease. As discussed in this review, there are marked differences between patients bearing distinct molecular subtypes. There is a new challenge with the intra-tumor heterogeneity that makes a particular tumor an assembly of a variety of subpopulations of cells surrounded by an interacting microenvironment. All of these factors complicate the diagnosis, therapy selection, and subsequent response. Therefore, it becomes crucial to understand all basic facets of tumor biology, genetic mutations, and epigenetic changes considered together for new approaches to cancer therapies and for their role in treatment resistance. Now in the "OMICS" era, we are beginning to understand the vast catalog of mutations and epigenetic changes at the subclone level. Thus, the future of modern oncology is towards a personalized medicine where the intra-tumor heterogeneity and the crosstalk between tumor cells and their microenvironment are being addressed.

\section{ACKNOWLEDGMENTS}

The authors thank Dr. Alberto Huberman, from the Biochemistry Unit of the Instituto Nacional de Ciencias Médicas y Nutrición SZ, Mexico City, for his critical review of the manuscript.

\section{REFERENCES}

1. Ferlay J, Soerjomataram I, Dikshit R, et al. Cancer incidence and mortality worldwide: sources, methods and major patterns in GLOBOCAN 2012. Int J Cancer. 2015;136:E359-86.

2. Torre LA, Siegel RL, Ward EM, Jemal A. Global cancer incidence and mortality rates and trends-An update. Cancer Epidemiol Biomarkers Prev. 2016;25:16-27.

3. Soto-Perez-de-Celis E, Chavarri-Guerra Y. National and regional breast cancer incidence and mortality trends in Mexico 20012011: Analysis of a population-based database. Cancer Epidemiol. 2016;41:24-33.

4. Sorlie T, Perou CM, Tibshirani R, et al. Gene expression patterns of breast carcinomas distinguish tumor subclasses with clinical implications. Proc Natl Acad Sci USA. 2001;98:10869-74.

5. Perou CM, Sorlie T, Eisen MB, et al. Molecular portraits of human breast tumours. Nature. 2000;406:747-52.

6. Hu Z, Fan C, Oh DS, et al. The molecular portraits of breast tumors are conserved across microarray platforms. BMC Genomics. 2006;7:96.

7. Cancer Genome Atlas N. Comprehensive molecular portraits of human breast tumours. Nature. 2012;490:61-70.

8. Yang WT, Zhu XZ. [The introduction of 2012 WHO classification of tumours of the breast]. Zhonghua Bing Li Xue Za Zhi. 2013;42:78-80.

9. Yeung C, Hilton J, Clemons M, et al. Estrogen, progesterone, and HER2/neu receptor discordance between primary and metastatic breast tumours-a review. Cancer Metastasis Rev. 2016; 35:427-37.

10. Almendro V, Marusyk A, Polyak K. Cellular heterogeneity and molecular evolution in cancer. Annu Rev Pathol. 2013;8:277-302.

11. McGranahan N, Swanton C. Biological and therapeutic impact of intratumor heterogeneity in cancer evolution. Cancer Cell. 2015;27:15-26.

12. Greaves M, Maley CC. Clonal evolution in cancer. Nature. 2012;481:306-13.

13. Meacham CE, Morrison SJ. Tumour heterogeneity and cancer cell plasticity. Nature. 2013;501:328-37.

14. Nowell PC. The clonal evolution of tumor cell populations. Science. 1976;194:23-8.

15. Burrell RA, McGranahan N, Bartek J, Swanton C. The causes and consequences of genetic heterogeneity in cancer evolution. $\mathrm{Na}$ ture. 2013;501:338-45.

16. Walter MJ, Shen D, Ding L, et al. Clonal architecture of secondary acute myeloid leukemia. N Engl J Med. 2012;366:1090-8.

17. Bolli N, Avet-Loiseau H, Wedge DC, et al. Heterogeneity of genomic evolution and mutational profiles in multiple myeloma. Nat Commun. 2014;5:2997.

18. Geyer FC, Weigelt B, Natrajan R, et al. Molecular analysis reveals a genetic basis for the phenotypic diversity of metaplastic breast carcinomas. J Pathol. 2010;220:562-73.

19. Turner NC, Reis-Filho JS. Genetic heterogeneity and cancer drug resistance. Lancet Oncol. 2012;13:e178-85.

20. Hiley C, de Bruin EC, McGranahan N, Swanton C. Deciphering intratumor heterogeneity and temporal acquisition of driver events to refine precision medicine. Genome Biol. 2014;15:453.

21. Gerlinger M, Swanton C. How Darwinian models inform therapeutic failure initiated by clonal heterogeneity in cancer medicine. $\mathrm{Br}$ J Cancer. 2010;103:1139-43. 
22. Yap TA, Gerlinger M, Futreal PA, Pusztai L, Swanton C. Intratumor heterogeneity: seeing the wood for the trees. Sci Transl Med. 2012;4:127ps10.

23. Miller WR, Larionov A. Changes in expression of oestrogen regulated and proliferation genes with neoadjuvant treatment highlight heterogeneity of clinical resistance to the aromatase inhibitor, letrozole. Breast Cancer Res. 2010;12:R52.

24. Miller CA, Gindin Y, Lu C, et al. Aromatase inhibition remodels the clonal architecture of estrogen-receptor-positive breast cancers. Nat Commun. 2016;7:12498.

25. Plaks V, Kong N, Werb Z. The cancer stem cell niche: how essential is the niche in regulating stemness of tumor cells? Cell Stem Cell. 2015;16:225-38.

26. Al-Haji M, Becker MW, Wicha M, Weissman I, Clarke MF. Therapeutic implications of cancer stem cells. Curr Opin Genet Dev. 2004;14:43-7.

27. Ponti D, Costa A, Zaffaroni N, et al. Isolation and in vitro propagation of tumorigenic breast cancer cells with stem/progenitor cell properties. Cancer Res. 2005;65:5506-11.

28. Medema JP. Cancer stem cells: the challenges ahead. Nat Cell Biol. 2013;15:338-44

29. Marjanovic ND, Weinberg RA, Chaffer CL. Cell plasticity and heterogeneity in cancer. Clin Chem. 2013;59:168-79.

30. Al-Hajj M, Wicha MS, Benito-Hernandez A, Morrison SJ, Clarke MF. Prospective identification of tumorigenic breast cancer cells. Proc Natl Acad Sci USA. 2003;100:3983-8.

31. Campos-Sanchez E, Cobaleda C. Tumoral reprogramming: Plasticity takes a walk on the wild side. Biochim Biophys Acta. 2015;1849:436-47.

32. Kalluri R, Weinberg RA. The basics of epithelial-mesenchymal transition. J Clin Invest. 2009;119:1420-8.

33. Gurzu S, Turdean S, Kovecsi A, Contac AO, Jung I. Epithelial-mesenchymal, mesenchymal-epithelial, and endothelial-mesenchymal transitions in malignant tumors: An update. World J Clin Cases. 2015;3:393-404.

34. Mani SA, Guo W, Liao MJ, et al. The epithelial-mesenchymal transition generates cells with properties of stem cells. Cell. 2008; 133:704-15.

35. Celia-Terrassa T, Meca-Cortes O, Mateo F, et al. Epithelial-mesenchymal transition can suppress major attributes of human epithelial tumor-initiating cells. J Clin Invest. 2012;122:1849-68.

36. Chaffer $\mathrm{CL}$, Marjanovic ND, Lee $\mathrm{T}$, et al. Poised chromatin at the ZEB1 promoter enables breast cancer cell plasticity and enhances tumorigenicity. Cell. 2013;154:61-74

37. Morel AP, Lievre M, Thomas C, Hinkal G, Ansieau S, Puisieux A. Generation of breast cancer stem cells through epithelial-mesenchymal transition. PLoS One. 2008;3:e2888.

38. Pinto CA, Widodo $E$, Waltham M, Thompson EW. Breast cancer stem cells and epithelial mesenchymal plasticity - Implications for chemoresistance. Cancer Lett. 2013;341:56-62.

39. Bednarz-Knoll N, Alix-Panabieres C, Pantel K. Plasticity of disseminating cancer cells in patients with epithelial malignancies. Cancer Metastasis Rev. 2012;31:673-87

40. Bonnomet A, Syne L, Brysse A, et al. A dynamic in vivo model of epithelial-to-mesenchymal transitions in circulating tumor cells and metastases of breast cancer. Oncogene. 2012;31: 3741-53.

41. Lim E, Vaillant F, Wu D, et al. Aberrant luminal progenitors as the candidate target population for basal tumor development in BRCA1 mutation carriers. Nat Med. 2009;15:907-13.

42. Marusyk A, Polyak K. Tumor heterogeneity: causes and consequences. Biochim Biophys Acta. 2010;1805:105-17

43. Marusyk A, Almendro V, Polyak K. Intra-tumour heterogeneity: a looking glass for cancer? Nat Rev Cancer. 2012;12:323-34.

44. Esparza-Lopez J, Ramos-Elias PA, Castro-Sanchez A, et al. Primary breast cancer cell culture yields intra-tumor heterogeneous subpopulations expressing exclusive patterns of receptor tyrosine kinases. BMC Cancer. 2016;16:740.

45. Gerlinger M, Rowan AJ, Horswell S, et al. Intratumor heterogeneity and branched evolution revealed by multiregion sequencing. N Engl J Med. 2012;366:883-92.

46. Navin N, Krasnitz A, Rodgers L, et al. Inferring tumor progression from genomic heterogeneity. Genome Res. 2010;20:68-80.

47. Navin N, Kendall J, Troge J, et al. Tumour evolution inferred by single-cell sequencing. Nature. 2011;472:90-4.

48. Hanahan D, Weinberg RA. Hallmarks of cancer: the next generation. Cell. 2011;144:646-74

49. Polyak K, Marusyk A. Cancer: Clonal cooperation. Nature. 2014;508:52-3.

50. Ashworth A, Lord C], Reis-Filho JS. Genetic interactions in cancer progression and treatment. Cell. 2011;145:30-8.
51. Shah SP Morin RD, Khattra J, et al Mutational evolution in a lobular breast tumour profiled at single nucleotide resolution. Nature. 2009;461:809-13.

52. Park SY, Gonen M, Kim HJ, Michor F, Polyak K. Cellular and genetic diversity in the progression of in situ human breast carcinomas to an invasive phenotype. J Clin Invest. 2010;120:636-44.

53. Shah SP, Roth A Goya R et al The clonal and mutational evolution spectrum of primary triple-negative breast cancers. Nature. 2012;486:395-9.

54. Kobayashi M, Ooi A, Oda Y, Nakanishi I. Protein overexpression and gene amplification of c-erbB-2 in breast carcinomas: a comparative study of immunohistochemistry and fluorescence in situ hybridization of formalin-fixed, paraffin-embedded tissues. Hum Pathol. 2002;33:21-8

55. Hanna W, Nofech-Mozes S, Kahn HJ. Intratumoral heterogeneity of HER2/neu in breast cancer--a rare event. Breast J. 2007;13:122-9.

56. Andersson J, Linderholm B, Bergh J, Elmberger G. HER-2/neu (c-erbB-2) evaluation in primary breast carcinoma by fluorescent in situ hybridization and immunohistochemistry with special focus on intratumor heterogeneity and comparison of invasive and in situ components. Appl Immunohistochem Mol Morphol. 2004; 12:14-20

57. Nadji M, Gomez-Fernandez C, Ganjei-Azar P, Morales AR. Immunohistochemistry of estrogen and progesterone receptors reconsidered: experience with 5,993 breast cancers. Am J Clin Pathol. 2005;123:21-7.

58. Taucher S, Rudas M, Gnant M, et al. Sequential steroid hormone receptor measurements in primary breast cancer with and without intervening primary chemotherapy. Endocr Relat Cancer. 2003;10:91-8

59. Bonsing BA, Corver WE, Fleuren GJ, Cleton-Jansen AM, Devilee $\mathrm{P}$, Cornelisse CJ. Allelotype analysis of flow-sorted breast cancer cells demonstrates genetically related diploid and aneuploid subpopulations in primary tumors and lymph node metastases. Genes Chromosomes Cancer. 2000;28:173-83.

60. Pandis N, Teixeira MR, Adeyinka A, et al. Cytogenetic comparison of primary tumors and lymph node metastases in breast cancer patients. Genes Chromosomes Cancer. 1998;22:122-9.

61. Kuukasjarvi T, Karhu R, Tanner M, et al. Genetic heterogeneity and clonal evolution underlying development of asynchronous metastasis in human breast cancer. Cancer Res. 1997;57:1597-604.

62. Nik-Zainal S, Van Loo P, Wedge DC, et al. The life history of 21 breast cancers. Cell. 2012;149:994-1007.

63. Keen JC, Yan L, Mack KM, et al. A novel histone deacetylase inhibitor, scriptaid, enhances expression of functional estrogen receptor alpha (ER) in ER negative human breast cancer cells in combination with 5-aza 2'-deoxycytidine. Breast Cancer Res Treat. 2003;81:177-86.

64. Fan J, Yin WJ, Lu JS, et al. ER alpha negative breast cancer cells restore response to endocrine therapy by combination treatment with both HDAC inhibitor and DNMT inhibitor. J Cancer Res Clin Oncol. 2008;134:883-90.

65. Wistuba, II, Gelovani JG, Jacoby JJ, Davis SE, Herbst RS. Methodological and practical challenges for personalized cancer therapies. Nat Rev Clin Oncol. 2011;8:135-41.

66. La Thangue NB, Kerr DJ. Predictive biomarkers: a paradigm shift towards personalized cancer medicine. Nat Rev Clin Oncol. 2011:8:587-96.

67. Oosterwijk JC, de Vries J, Mourits MJ, de Bock GH. Genetic testing and familial implications in breast-ovarian cancer families. Maturitas. 2014:78:252-7.

68. Stefansson OA, Esteller M. Epigenetic modifications in breast cancer and their role in personalized medicine. Am J Pathol. 2013;183:1052-63.

69. Arrowsmith $\mathrm{CH}$, Bountra C, Fish PV, Lee K, Schapira M. Epigenetic protein families: a new frontier for drug discovery. Nat Rev Drug Discov. 2012;11:384-400.

70. Bestor TH, Bourc'his D. Genetics and epigenetics of hydatidiform moles. Nat Genet. 2006;38:274-6.

71. Baylin SB, Jones PA. A decade of exploring the cancer epigenome - biological and translational implications. Nat Rev Cancer. 2011;11:726-34

72. Korber P, Becker PB. Nucleosome dynamics and epigenetic stability. Essays Biochem. 2010;48:63-74.

73. Thurman RE, Day N, Noble WS, Stamatoyannopoulos JA. Identification of higher-order functional domains in the human ENCODE regions. Genome Res. 2007;17:917-27

74. Hansen KD, Timp W, Bravo HC, et al. Increased methylation variation in epigenetic domains across cancer types. Nat Genet. 2011;43:768-75

75. Berman BP, Weisenberger DJ, Aman JF, et al. Regions of foca DNA hypermethylation and long-range hypomethylation in 
colorectal cancer coincide with nuclear lamina-associated domains. Nat Genet. 2011;44:40-6.

76. Hon GC, Hawkins RD, Caballero OL, et al. Global DNA hypomethylation coupled to repressive chromatin domain formation and gene silencing in breast cancer. Genome Res. 2012;22:246-58.

77. Hiken JF, McDonald JI, Decker KF, et al. Epigenetic activation of the prostaglandin receptor EP4 promotes resistance to endocrine therapy for breast cancer. Oncogene. 2016. [Epub ahead of print].

78. You JS, Jones PA. Cancer genetics and epigenetics: two sides of the same coin? Cancer Cell. 2012;22:9-20.

79. Fanale D, Amodeo V, Corsini LR, Rizzo S, Bazan V, Russo A. Breast cancer genome-wide association studies: there is strength in numbers. Oncogene. 2012;31:2121-8.

80. Esteller M. Epigenetics in cancer. N Engl J Med. 2008;358:1148-59.

81. Herman JG, Latif F, Weng $Y$, et al. Silencing of the VHL tumor-suppressor gene by DNA methylation in renal carcinoma. Proc Natl Acad Sci USA. 1994;91:9700-4.

82. Mitra D, Das PM, Huynh FC, Jones FE. Jumonji/ARID1 B (JARID1B) protein promotes breast tumor cell cycle progression through epigenetic repression of microRNA let-7e. J Biol Chem. 2011;286:40531-5.

83. Stephens PJ, Tarpey PS, Davies H, et al. The landscape of cancer genes and mutational processes in breast cancer. Nature. 2012;486:400-4

84. Dobrovic A, Simpfendorfer D. Methylation of the BRCA1 gene in sporadic breast cancer. Cancer Res. 1997;57:3347-50.

85. Birgisdottir V, Stefansson OA, Bodvarsdottir SK, Hilmarsdottir $\mathrm{H}$, Jonasson JG, Eyfjord JE. Epigenetic silencing and deletion of the BRCA1 gene in sporadic breast cancer. Breast Cancer Res. 2006;8:R38.

86. Garcia JM, Silva J, Pena C, et al. Promoter methylation of the PTEN gene is a common molecular change in breast cancer. Genes Chromosomes Cancer. 2004;41:117-24.

87. Woodcock DM, Linsenmeyer ME, Doherty JP, Warren WD. DNA methylation in the promoter region of the p16 (CDKN2/MTS-1/ INK4A) gene in human breast tumours. Br J Cancer. 1999;79:251-6.

88. Holm K, Hegardt C, Staaf J, et al. Molecular subtypes of breast cancer are associated with characteristic DNA methylation patterns. Breast Cancer Res. 2010;12:R36.

89. Lawrence MS, Stojanov P, Polak P, et al. Mutational heterogeneity in cancer and the search for new cancer-associated genes. $\mathrm{Na}$ ture. 2013;499:214-8.

90. Barekati Z, Radpour R, Kohler C, et al. Methylation profile of TP53 regulatory pathway and mtDNA alterations in breast cancer patients lacking TP53 mutations. Hum Mol Genet. 2010;19:2936-46.

91. Prabhu JS, Wahi K, Korlimarla A, et al. The epigenetic silencing of the estrogen receptor (ER) by hypermethylation of the ESR1 promoter is seen predominantly in triple-negative breast cancers in Indian women. Tumour Biol. 2012;33:315-23.

92. Wu L, Wang F, Xu R, et al. Promoter methylation of BRCA1 in the prognosis of breast cancer: a meta-analysis. Breast Cancer Res Treat. 2013;142:619-27.

93. Cho YH, Yazici H, Wu HC, et al. Aberrant promoter hypermethylation and genomic hypomethylation in tumor, adjacent normal tissues and blood from breast cancer patients. Anticancer Res. 2010;30:2489-96

94. Hesson LB, Cooper WN, Latif F. The role of RASSF1A methylation in cancer. Dis Markers. 2007;23:73-87.

95. Collins N, Wooster R, Stratton MR. Absence of methylation of CpG dinucleotides within the promoter of the breast cancer susceptibility gene BRCA2 in normal tissues and in breast and ovarian cancers. Br J Cancer. 1997;76:1150-6.

96. Bal A, Verma S, Joshi K, et al. BRCA1-methylated sporadic breast cancers are BRCA-like in showing a basal phenotype and absence of ER expression. Virchows Arch. 2012;461:305-12.

97. Bissell MJ, Hines WC. Why don't we get more cancer? A proposed role of the microenvironment in restraining cancer progression. Nat Med. 2011;17:320-9.

98. Wang CC, Bajikar SS, Jamal L, Atkins KA, Janes KA. A time- and matrix-dependent TGFBR3-JUND-KRT5 regulatory circuit in single breast epithelial cells and basal-like premalignancies. Nat Cell Biol. 2014;16:345-56.

99. Bussard KM, Mutkus L, Stumpf K, Gomez-Manzano C, Marini FC. Tumor-associated stromal cells as key contributors to the tumor microenvironment. Breast Cancer Res. 2016;18:84.

100. Castano Z, Fillmore CM, Kim CF, McAllister SS. The bed and the bugs: interactions between the tumor microenvironment and cancer stem cells. Semin Cancer Biol. 2012;22:462-70.

101. Dunn GP, Bruce AT, Ikeda H, Old LJ, Schreiber RD. Cancer immunoediting: from immunosurveillance to tumor escape. Nat Immunol. 2002;3:991-8.
102. Shevach EM. Fatal attraction: tumors beckon regulatory $T$ cells. Nat Med. 2004;10:900-1.

103. Demaria S, Volm MD, Shapiro RL, et al. Development of tumor-infiltrating lymphocytes in breast cancer after neoadjuvant paclitaxel chemotherapy. Clin Cancer Res. 2001;7:3025-30.

104. Denkert C, Loibl S, Noske A, et al. Tumor-associated lymphocytes as an independent predictor of response to neoadjuvant chemotherapy in breast cancer. J Clin Oncol. 2010;28:105-13.

105. Cimino-Mathews A, Foote JB, Emens LA. Immune targeting in breast cancer. Oncology (Williston Park). 2015;29:375-85.

106. Lehmann BD, Bauer JA, Chen X, et al. Identification of human triple-negative breast cancer subtypes and preclinical models for selection of targeted therapies. J Clin Invest. 2011;121: 2750-67.

107. Emens LA. Breast cancer immunobiology driving immunotherapy: vaccines and immune checkpoint blockade. Expert Rev Anticancer Ther. 2012;12:1597-611

108. Pardoll DM. The blockade of immune checkpoints in cancer immunotherapy. Nat Rev Cancer. 2012;12:252-64.

109. Balkwill F, Mantovani A. Inflammation and cancer: back to Virchow? Lancet. 2001:357:539-45.

110. Pierce BL, Ballard-Barbash R, Bernstein L, et al, et al. Elevated biomarkers of inflammation are associated with reduced survival among breast cancer patients. J Clin Oncol. 2009;27:3437-44

111. Conze D, Weiss L, Regen PS et al. Autocrine production of interleukin 6 causes multidrug resistance in breast cancer cells. Cancer Res. 2001;61:8851-8.

112. Sullivan NJ, Sasser AK, Axel AE, et al. Interleukin-6 induces an epithelial-mesenchymal transition phenotype in human breast cancer cells. Oncogene. 2009;28:2940-7.

113. Esparza-Lopez J, Medina-Franco H, Escobar-Arriaga E, et al Doxorubicin induces atypical NF-kappaB activation through c-Abl kinase activity in breast cancer cells. J Cancer Res Clin Oncol. 2013;139:1625-35.

114. Wu Y, Deng J, Rychahou PG, Oiu S, Evers BM, Zhou BP. Stabilization of snail by NF-kappaB is required for inflammation-induced cell migration and invasion. Cancer Cell. 2009;15:416-28.

115. Pouliot MC, Labrie Y, Diorio C, Durocher F. The role of methylation in breast cancer susceptibility and treatment. Anticancer Res. 2015;35:4569-74.

116. Creighton CJ, Li X, Landis M, et al. Residual breast cancers after conventional therapy display mesenchymal as well as tumor-initiating features. Proc Natl Acad Sci USA. 2009;106:13820-5.

117. Li QQ, Xu JD, Wang WJ, et al. Twist1-mediated adriamycin-induced epithelial-mesenchymal transition relates to multidrug resistance and invasive potential in breast cancer cells. Clin Cancer Res. 2009;15:2657-65.

118. Kajita M, McClinic KN, Wade PA. Aberrant expression of the transcription factors snail and slug alters the response to genotoxic stress. Mol Cell Biol. 2004;24:7559-66

119. Tryndyak VP, Beland FA, Pogribny IP. E-cadherin transcriptional down-regulation by epigenetic and microRNA-200 family alterations is related to mesenchymal and drug-resistant phenotypes in human breast cancer cells. Int J Cancer. 2010;126:2575-83.

120. DeNardo DG, Brennan DJ, Rexhepaj E, et al. Leukocyte complexity predicts breast cancer survival and functionally regulates response to chemotherapy. Cancer Discov. 2011;1:54-67.

121. Yang R, Pfutze K, Zucknick M, et al. DNA methylation array analyses identified breast cancer-associated HYAL2 methylation in peripheral blood. Int J Cancer. 2015;136:1845-55.

122. Guerrero-Preston R, Hadar T, Ostrow KL, et al. Differential promoter methylation of kinesin family member $1 \mathrm{a}$ in plasma is associated with breast cancer and DNA repair capacity. Oncol Rep. 2014;32:505-12

123. Heyn H, Carmona FJ, Gomez A, et al. DNA methylation profiling in breast cancer discordant identical twins identifies DOK7 as novel epigenetic biomarker. Carcinogenesis. 2013;34:102-8.

124. Santos-Reboucas CB, Pimentel MM. Implication of abnormal epigenetic patterns for human diseases. Eur J Hum Genet. 2007;15:10-7.

125. Fong PC, Boss DS, Yap TA, et al. Inhibition of poly(ADP-ribose) polymerase in tumors from BRCA mutation carriers. $N$ Engl ] Med. 2009;361:123-34

126. Widschwendter M, Siegmund KD, Muller HM, et al. Association of breast cancer DNA methylation profiles with hormone receptor status and response to tamoxifen. Cancer Res. 2004;64:3807-13.

127. Farmer H, McCabe N, Lord C], et al. Targeting the DNA repair defect in BRCA mutant cells as a therapeutic strategy. Nature. 2005;434:917-21.

128. Patel AG, De Lorenzo SB, Flatten KS, Poirier GG, Kaufmann SH. Failure of iniparib to inhibit poly(ADP-Ribose) polymerase in vitro. Clin Cancer Res. 2012;18:1655-62. 